\title{
Restricted basis set coupled-channel calculations on atom-molecule collisions in magnetic fields
}

\author{
Masato Morita ${ }^{1}$ and Timur V. Tscherbul ${ }^{1}$ \\ ${ }^{1}$ Department of Physics, University of Nevada, Reno, NV, 8955\%, USA*
}

(Dated: October 8, 2018)

\begin{abstract}
Rigorous coupled-channel quantum scattering calculations on molecular collisions in external fields are computationally demanding due to the need to account for a large number of coupled channels and multiple total angular momenta $J$ of the collision complex. We show that by restricting the number of total angular momentum basis states to include only the states with helicities $K \leq K_{\max }$ it is possible to obtain accurate elastic and inelastic cross sections for $\mathrm{He}+\mathrm{CaH}$, $\mathrm{Li}+\mathrm{CaH}$ and $\mathrm{Li}+\mathrm{SrOH}$ collisions at a small fraction of the computational cost of the full coupled-channel calculations (where $K$ is the projection of the molecular rotational angular momentum on the atom-diatom axis). The optimal size of the truncated helicity basis set depends on the mechanism of the inelastic process and on the magnitude of the external magnetic field. For dipolar-mediated spin relaxation in ultracold $\mathrm{Li}+\mathrm{CaH}$ and $\mathrm{Li}+\mathrm{SrOH}$ collisions, we find that a minimal helicity basis set $\left(K_{\max }=0\right)$ gives quantitatively accurate results at ultralow collision energies, leading to nearly 90-fold gain in computational efficiency. Larger basis sets are required to accurately describe the resonance structure in $\mathrm{Li}+\mathrm{CaH}$ and $\mathrm{Li}+\mathrm{SrOH}$ inelastic cross sections in the few partial wave-regime $\left(K_{\max }=3\right)$ as well as indirect spin relaxation in $\mathrm{He}+\mathrm{CaH}$ collisions $\left(K_{\max }=1\right)$. Our calculations indicate that the resonance structure is due to an interplay of the spin-rotation and Coriolis couplings between the basis states of different $K$ and the couplings between the rotational states of the same $K$ induced by the anisotropy of the interaction potential.
\end{abstract}




\section{INTRODUCTION}

Steady experimental advances in molecular cooling and trapping $\underline{1}^{-\frac{5}{n}}$ have led to the production of trapped ensembles of molecules in precisely defined internal states at temperatures below $1 \mathrm{~K}$, which are being actively used to study molecular collision dynamics at ultralow temperatures $\underline{\underline{6}-\underline{-8}}$. These studies explore the new regimes of quantum dynamics intrinsic to low-temperature molecular scattering, including Wigner threshold laws, scattering resonances $\underline{\underline{9}} \underline{-11}$, and tunnelling $\underline{12}-\underline{14}$. Another remarkable aspect of ultracold molecular collisions is the capability to control their outcome with external electromagnetic fields $\mathbf{4}, 13-20$.

A rigorous theoretical description of two-body collisions in ultracold molecular gases in the presence of external fields is essential for interpreting the results of experimental measurements on trapped molecules and atom-molecule mixtures $\underline{4} \underline{20}$. While elastic collisions between trapped molecules are desirable as they lead to thermalization, inelastic collisions release the energy stored in the molecule's internal degrees of freedom, thereby reducing the efficiency of sympathetic and evaporative cooling of molecular gases 4,20 . It is therefore essential to be able to estimate the low-temperature collisional properties of molecular gases and atom-molecule mixtures from first principles based on the solution of multichannel Schrödinger equation for the collision complex $\underline{4,13,14,20}$.

The numerical solution of the multichannel Schrödinger equation for molecular collisions in the presence of external fields poses a challenging computational problem due to an extremely large density of states inherent to most molecular collision complexes $20-24$. This problem arises due to the strong and anisotropic intermolecular interactions, which couple a large number of low-energy rotational channels $20-24$. Taking advantage of the symmetry of the collision complex by using the eigenstates of its total angular momentum as a basis provides an efficient way to handle the anisotropic interactions, leading to a dramatic reduction in the number of scattering channels 20,25 . The ensuing reduction of the computational cost has opened up the possibility to perform converged coupled-channel (CC) calculations on heavy, strongly anisotropic atom-molecule collisions and chemical reactions in the presence of external magnetic $\underline{22}^{24}, \underline{26}$ and electric $\underline{14}, \underline{27}$ fields. However, such calculations still involve thousands of scattering channels, calling for the development of new methods and approximations to further reduce the number of scattering basis functions.

The use of incomplete helicity basis sets is one such approximation, which takes advan- 
tage of an approximate conservation of molecular helicity $K$, the projection of the molecule's rotational angular momentum on the atom-molecule axis $\underline{28} \underline{-31}$. This near-conservation ensures that only a limited number of $K$ values make a non-negligible contribution to the scattering process, and hence the basis set can be truncated at $K=K_{\max }$ without compromising the quality of the results. Restricted helicity basis sets are commonly used in field-free quantum CC calculations of inelastic collisions $\underline{30}$ and chemical reactions $\underline{28,29,32}$ of closed-shell diatomic molecules with structureless atoms. We note that for chemical reactions that are not dominated by near-collinear transition states such as $\mathrm{Li}+\mathrm{HF} \rightarrow \mathrm{LiF}+\mathrm{H}$, such basis sets provide no computational advantage, and complete helicity basis sets should be used to obtain quantitatively accurate results 31 .

A related approximate technique, which retains all values of $K$ in the basis set but neglects the Coriolis coupling between them, is known as the helicity-conserving coupled-states (CS) approximation $\underline{3} \underline{34}$. The CS approximation generally provides quantitatively accurate results for molecular collisions at room temperature and above $\underline{\underline{33}} \underline{\underline{36}}$. The CS approximation has also been tested for cold molecular collisions and reactions in the absence of external fields and found to produce accurate results $\frac{36}{37}$ for the background (non-resonant) reactive scattering of $\mathrm{HCl}$ and $\mathrm{DCl}$ molecules in their ground rotational states with $\mathrm{F}$ atoms. However, neither helicity truncation nor CS approximation have been applied to molecular collisions in the presence of external electromagnetic fields.

Here, we explore the use of restricted helicity basis sets in quantum scattering calculations on ultracold atom-molecule collisions in the presence of an external magnetic field. We focus on an important class of inelastic collisions that change the internal spin (Zeeman) state of the colliding molecules known as spin relaxation. We show that quantum scattering calculations employing reduced helicity basis sets provide accurate results for the elastic and spin relaxation cross sections in the resonance-free regime over a wide range of magnetic fields and collision energies at a reduced computational cost.

This paper is organized as follows. In Sec. II we outline the theoretical methodology for rigorous atom-molecule $\mathrm{CC}$ calculations in a magnetic field and describe the procedure of basis set truncation. In Sec. III we compare the approximate results obtained using truncated basis sets with the full $\mathrm{CC}$ calculations for low-temperature $\mathrm{He}+\mathrm{CaH}, \mathrm{Li}+\mathrm{CaH}$, and $\mathrm{Li}+\mathrm{SrOH}$ collisions in a magnetic field. We analyze the performance of the different $K$ basis sets and estimate the computational speedups achieved. In Sec. IIIB, we examine 
the resonance structure in $\mathrm{Li}+\mathrm{CaH}$ inelastic cross sections at high magnetic fields as an example of the situation where truncated basis calculations do not provide an accurate approximation to the full CC results. Sec. IV concludes with a brief summary of results and outlines directions for future research.

\section{THEORY}

In this section we first outline the rigorous $\mathrm{CC}$ methodology for atom-molecule collisions in the presence of an external magnetic field ${ }^{25}$ and then describe the procedure of helicity basis set truncation. Throughout this work we will be concerned with non-reactive collisions of diatomic molecules in the electronic states of ${ }^{2} \Sigma$ symmetry with atoms in the ${ }^{1} \mathrm{~S}$ and ${ }^{2} \mathrm{~S}$ electronic states. Examples include collisions of $\mathrm{He}\left({ }^{1} \mathrm{~S}\right)$ atoms with $\mathrm{CaH}\left({ }^{2} \Sigma\right)$ molecules, $\mathrm{Li}\left({ }^{2} \mathrm{~S}\right)$ atoms with $\mathrm{CaH}$ and $\mathrm{SrOH}\left({ }^{2} \Sigma\right)$ molecules, and $\mathrm{Rb}\left({ }^{2} \mathrm{~S}\right)$ atoms with $\operatorname{SrF}\left({ }^{2} \Sigma\right)$ molecules. Previous theoretical studies have found low inelastic collision rates in these systems, suggesting favorable prospects for sympathetic cooling of $\mathrm{CaH}, \mathrm{SrOH}$, and $\mathrm{SrF}$ molecular radicals by ultracold alkali-metal atoms in a magnetic trap $\underline{22,24,26}$.

The Hamiltonian of the atom-molecule collision complex is conveniently expressed in a body-fixed $(\mathrm{BF})$ coordinate frame with the $z$-axis defined by the atom-molecule Jacobi vector $\boldsymbol{R}$ and the $y$-axis perpendicular to the collision plane $e^{25,38,39}$

$$
\hat{H}=-\frac{1}{2 \mu R} \frac{\partial^{2}}{\partial R^{2}} R+\frac{1}{2 \mu R^{2}}\left(\hat{\boldsymbol{J}}-\hat{\boldsymbol{N}}-\hat{\boldsymbol{S}}_{a}-\hat{\boldsymbol{S}}\right)^{2}+\hat{V}(R, \theta)+\hat{H}_{\mathrm{at}}+\hat{H}_{\mathrm{mol}},
$$

where $\boldsymbol{r}$ is the internuclear Jacobi vector of the diatomic molecule, $R=|\boldsymbol{R}|, r=|\boldsymbol{r}|$, and $\theta$ is the angle between $\boldsymbol{R}$ and $\boldsymbol{r}$. In Eq. (1), $\hat{\boldsymbol{S}}_{a}$ and $\hat{\boldsymbol{S}}$ stand for the electron spins of the atom and the molecule, $\mu$ and $\hat{\boldsymbol{J}}$ are the reduced mass and the total angular momentum of the atom-molecule collision complex, $\boldsymbol{N}$ is the rotational angular momentum of the diatomic molecule (see below) and $\hat{V}(\boldsymbol{R}, \boldsymbol{r})$ is the atom-molecule interaction potential, including both the electrostatic and magnetic dipole-dipole interactions (for $S_{a} \neq 0$ ).

The asymptotic Hamiltonian $\hat{H}_{\text {as }}=\hat{H}_{\text {at }}+\hat{H}_{\text {mol }}$ describes the threshold structure of the collision complex in the limit $R \rightarrow \infty$. The molecular Hamiltonian $\hat{H}_{\text {mol }}$ defines the energy levels of the ${ }^{2} \Sigma$ diatomic molecule in its electronic and vibrational ground states in the presence of an external magnetic field $B^{40,41}$

$$
\hat{H}_{\mathrm{mol}}=B_{e} \hat{\boldsymbol{N}}^{2}+\gamma \hat{\boldsymbol{N}} \cdot \hat{\boldsymbol{S}}+2 \mu_{0} B \hat{S}_{Z}
$$


where $B_{e}$ is the rotational constant of the molecule, $\hat{\mathbf{N}}$ is the rotational angular momentum, $\hat{\boldsymbol{S}}$ is the electron spin, $\hat{S}_{Z}$ is the projection of $\hat{\boldsymbol{S}}$ on the magnetic field axis (the space-fixed (SF) quantization axis), $\mu_{0}$ is the Bohr magneton, and $\gamma$ is the spin-rotation constant $\underline{41}$. In what follows we will assume that the internuclear distance in the diatomic molecule $r$ is fixed at its equilibrium value $r_{e}$, which is a good approximation if the atom-molecule interaction potential $\hat{V}(R, \theta)$ depends on $r$ only weakly (true for He-CaH ${ }^{42}$ and alkali-SrF interactions ${ }^{43}$ ). Finally, the Hamiltonian of the ${ }^{2} \mathrm{~S}$ atom is given by (neglecting the hyperfine structure)

$$
\hat{H}_{\mathrm{at}}=2 \mu_{0} B \hat{S}_{a_{Z}}
$$

where the operator $\hat{S}_{a_{Z}}$ gives the projection of $\hat{S}_{a}$ on the SF quantization axis. Equation (2) represents the simplest possible Hamiltonian for an open-shell molecular radical; however, additional spin-dependent terms may be added to it as necessary to account for the electronic states of ${ }^{3} \Sigma$ or ${ }^{2} \Pi$ symmetries ${ }^{25} \underline{44}$ or the hyperfine structure ${ }^{25}, \underline{45}, 46$.

To solve the time-independent Schrödinger equation with the Hamiltonian (1) we expand the wavefunction of the atom-molecule collision complex in BF basis functions $25,38,39$

$$
\begin{gathered}
|\Psi\rangle=\frac{1}{R} \sum_{\alpha, J, \Omega} F_{\alpha J \Omega}^{M}(R)|\alpha\rangle|J M \Omega\rangle, \\
|\alpha\rangle=|N K\rangle|S \Sigma\rangle\left|S_{a} \Sigma_{a}\right\rangle,
\end{gathered}
$$

where $\Omega, K, \Sigma$, and $\Sigma_{a}$ are the projections of $\hat{J}, \hat{N}, \hat{S}$, and $\hat{S}_{a}$ on the BF quantization axis $z$ (denoted collectively by $\alpha) ; \Omega=K+\Sigma+\Sigma_{a}$. The Wigner $D$-functions $|J M \Omega\rangle=$ $\sqrt{(2 J+1) / 8 \pi^{2}} D_{M K}^{J *}(\hat{\Omega})$ depend on the Euler angles which specify the position of BF axes $x$, $y$, and $z$ in the SF frame. The functions $|N K\rangle=\sqrt{2 \pi} Y_{N K}(\theta, 0),|S \Sigma\rangle$, and $\left|S_{a} \Sigma_{a}\right\rangle$ describe the orientation of the diatomic molecule and the spin degrees of freedom in the BF frame.

The radial expansion coefficients $F_{\alpha J \Omega}^{M}(R)$, which carry information about scattering observables, may be found by solving the CC equations ${ }^{25}$

$$
\begin{aligned}
{\left[\frac{d^{2}}{d R^{2}}+2 \mu E\right] F_{\alpha J \Omega}^{M}(R)=2 \mu \sum_{\alpha^{\prime}, J,^{\prime} \Omega^{\prime}}\langle\alpha J \Omega| \hat{V}(R, \theta) } & +\frac{1}{2 \mu R^{2}}\left(\hat{\boldsymbol{J}}-\hat{\boldsymbol{N}}-\hat{\boldsymbol{S}}-\hat{\boldsymbol{S}}_{a}\right)^{2} \\
& +\hat{H}_{\mathrm{mol}}+\hat{H}_{\mathrm{at}}\left|\alpha^{\prime} J^{\prime} \Omega^{\prime}\right\rangle F_{\alpha^{\prime} J^{\prime} \Omega^{\prime}}^{M}(R),
\end{aligned}
$$

where $E$ is the total energy. The matrix elements of the interaction potential, orbital angular momentum and the asymptotic Hamiltonian on the right-hand side of Eq. (6) can 
be evaluated as described in our previous work $\mathrm{w}^{25,26}$. We use the accurate spectroscopic constants of $\mathrm{CaH}\left({ }^{2} \Sigma^{+}\right)$and $\operatorname{SrOH}\left({ }^{2} \Sigma^{+}\right)$and the most recent ab initio interaction potentials for $\mathrm{He}-\mathrm{CaH}^{42}$, $\mathrm{Li}_{-} \mathrm{CaH}^{22}$, and $\mathrm{Li}-\mathrm{SrOH}^{24}$. The size of the basis set is controlled by the truncation parameters $J_{\max }$ and $N_{\max }$, which give the maximum values of the total angular momentum $J$ and rotational angular momentum $N$ in the basis set.

We integrate the $\mathrm{CC}$ equations using the modified log-derivative method $\stackrel{47}{ }$ to obtain the log-derivative of the multichannel scattering wavefunction in the asymptotic region, where the atom-molecule interaction potential is negligible compared to the centrifugal potential and to the collision energy. The log-derivative matrix is then transformed to the SF representation and to a basis, which diagonalizes the asymptotic Hamiltonian ${ }^{25}$. As a final step, matching the transformed log-derivative matrix to the Riccati-Bessel functions and their derivatives $25 \underline{48}$ yields the $S$-matrix elements, from which the scattering cross sections are obtained using standard expressions ${ }^{25}$. The calculated scattering cross sections are converged to $<10 \%$ with respect to the basis set and radial grid parameters. Table I lists the values of the convergence parameters for the atom-molecule collision systems studied in this work.

To describe truncated helicity basis sets, we introduce an additional parameter $K_{\max }$, which gives the maximum value of the helicity in the BF basis (5). The maximum possible value of $K_{\max }$ is given by $\min \left(N_{\max }, J_{\max }+S+S_{a}\right)$, which corresponds to full CC calculations. Table II lists the numbers of BF basis functions for the atom-molecule collision systems studied here as a function of $K_{\max }$. The size of the basis set decreases substantially with decreasing $K_{\max }$, leading to a substantial reduction of the number of scattering channels at low $K_{\max }$. Since the computational cost of CC calculations scales as $N^{3}$ with the number of scattering channels $N$, using basis sets with small $K_{\max }$ can result in nearly two orders of magnitude reduction of the computational cost.

A priori, it is not clear whether truncation of the full helicity basis set can lead to quantitatively accurate results for low-temperature atom-molecule collisions in a magnetic field. Indeed, limiting the number of $K$-states in the basis set affects the matrix elements of $K$ dependent interactions in the molecular Hamiltonian, such as the spin-rotation interaction ${ }^{25}$

$$
\begin{aligned}
& \left\langle N K\left|\left\langle S \Sigma|\gamma \hat{\boldsymbol{N}} \cdot \hat{\boldsymbol{S}}| N^{\prime} K^{\prime}\right\rangle\right| S \Sigma^{\prime}\right\rangle=\delta_{N N^{\prime}}\left[\gamma_{\mathrm{SR}} K \Sigma \delta_{K K^{\prime}} \delta_{\Sigma \Sigma^{\prime}}\right. \\
& \left.\quad+\frac{\gamma_{\mathrm{SR}}}{2}\left[N^{\prime}\left(N^{\prime}+1\right)-K^{\prime}\left(K^{\prime} \pm 1\right)\right]^{1 / 2}\left[S(S+1)-\Sigma^{\prime}\left(\Sigma^{\prime} \mp 1\right)\right]^{1 / 2} \delta_{K, K^{\prime} \pm 1} \delta_{\Sigma, \Sigma^{\prime} \mp 1}\right]
\end{aligned}
$$


the atom-molecule interaction potential

$$
\begin{aligned}
& \left\langle J M \Omega\left|\left\langle N K\left|\left\langle S \Sigma|V(R, \theta)| J^{\prime} M^{\prime} \Omega^{\prime}\right\rangle\right| N^{\prime} K^{\prime}\right\rangle\right| S \Sigma^{\prime}\right\rangle=\delta_{J J^{\prime}} \delta_{M M^{\prime}} \delta_{\Sigma \Sigma^{\prime}} \\
& \quad \times\left[(2 N+1)\left(2 N^{\prime}+1\right)\right]^{1 / 2}(-1)^{K} \sum_{\lambda} V_{\lambda}(R)\left(\begin{array}{ccc}
N & \lambda & N^{\prime} \\
-K & 0 & K^{\prime}
\end{array}\right)\left(\begin{array}{ccc}
N & \lambda & N^{\prime} \\
0 & 0 & 0
\end{array}\right),
\end{aligned}
$$

and the squared orbital angular momentum of the atom-molecule complex

$$
\begin{aligned}
& \left\langle J M \Omega\left|\left\langle N K\left|\left\langle S \Sigma\left|(\hat{\boldsymbol{J}}-\hat{\boldsymbol{N}}-\hat{\boldsymbol{S}})^{2}\right| J^{\prime} M^{\prime} \Omega^{\prime}\right\rangle\right| N^{\prime} K^{\prime}\right\rangle\right| S \Sigma^{\prime}\right\rangle=\delta_{J J^{\prime}} \delta_{M M^{\prime}} \delta_{N N^{\prime}} \\
& \times\left[\mathcal{C}_{1}(J, \Omega, \alpha) \delta_{\Omega \Omega^{\prime}} \delta_{K K^{\prime}} \delta_{\Sigma \Sigma^{\prime}}-\mathcal{C}_{2}\left(J, \Omega, \Omega^{\prime}, \alpha, \alpha^{\prime}\right) \delta_{\Omega \Omega^{\prime}} \delta_{\Omega, \Omega^{\prime} \pm 1} \delta_{K, K^{\prime} \pm 1} \delta_{\Sigma \Sigma^{\prime}}\right. \\
& \left.\quad-\mathcal{C}_{3}\left(J, \Omega, \Omega^{\prime}, \alpha, \alpha^{\prime}\right) \delta_{\Omega, \Omega^{\prime} \pm 1} \delta_{K, K^{\prime}} \delta_{\Sigma, \Sigma^{\prime} \pm 1}-\mathcal{C}_{4}\left(\alpha, \alpha^{\prime}\right) \delta_{\Omega \Omega^{\prime}} \delta_{K, K^{\prime} \pm 1} \delta_{\Sigma, \Sigma^{\prime} \mp 1}\right]
\end{aligned}
$$

where $\mathcal{C}_{i}$ are angular momentum coefficients that generally depend on $J, \Omega, N, \Sigma$, and $K$ (see Ref. 25 for explicit expressions). Note that the spin-rotation interaction (Eq. 7) as well as the centrifugal term (Eq. 9) couple the basis functions with $K^{\prime}=K$ and $K^{\prime}=K \pm 1$ whereas the atom-molecule interaction potential (Eq. 8) is diagonal in $K \underline{25}$

The above expressions suggest that truncation of the helicity basis is expected to produce quantitatively accurate results if the matrix elements of the spin-rotation interaction, the atom-molecule interaction potential, and of the centrifugal term omitted from the basis are much smaller in absolute magnitude than the matrix elements retained in the basis. In the following we will examine the effect of basis set truncation on the scattering observables for ultracold $\mathrm{He}+\mathrm{CaH}, \mathrm{Li}+\mathrm{CaH}$, and $\mathrm{Li}+\mathrm{SrOH}$ collisions in a magnetic field.

\section{RESULTS AND DISCUSSION}

\section{A. $\mathrm{He}+\mathrm{CaH}$ collisions}

In this section, we present the results of restricted basis set $\mathrm{CC}$ calculations of the elastic and spin relaxation cross sections for $\mathrm{He}+\mathrm{CaH}$ collisions in the presence of an external magnetic field. $\mathrm{He}+\mathrm{CaH}$ is a prototype weakly anisotropic collision system explored in a number of previous theoretical studies $\frac{17,25,49,50}{2}$ using rigorous CC methods and an accurate ab initio PES. These theoretical calculations were in semi-quantitative agreement with ex-

perimental measurements of collision-induced spin relaxation rates of magnetically trapped $\mathrm{CaH}$ molecules at $0.5 \mathrm{~K}^{51}$. 
Figure 1(a) shows the cross sections for elastic scattering and inelastic relaxation in $\mathrm{He}+\mathrm{CaH}$ collisions as a function of the collision energy. We focus on the spin-flipping transition $M_{S}=1 / 2 \rightarrow M_{S}=-1 / 2$ in collisions of $\mathrm{CaH}\left({ }^{2} \Sigma\right)$ molecules within the ground rotational state, which was the subject of several experimental 51,52 and many theoretical $17,25,49,50$ studies. Here, $M_{S}$ is the projection of the molecular spin onto the magnetic field axis.

The inelastic cross sections are 6 orders of magnitude smaller than the elastic cross section shown in the inset of Fig. 1 and they decrease with decreasing collision energy before reaching the Wigner threshold regime ${ }^{25} \underline{49} \sigma_{\text {inel }} \propto E^{-1 / 2}$. We observe that the inelastic cross sections computed with $K_{\max }=1$ are in excellent agreement with exact CC results to within $1 \%$. In contrast, restricted basis set results obtained with $K_{\max }=0$ underestimate the exact cross sections by more than 5 orders of magnitude. As shown below, this striking difference is due to a peculiar mechanism of spin relaxation in $\mathrm{He}+\mathrm{CaH}$ collisions. The elastic cross sections agree well with full CC calculations for both $K_{\max }=0$ and 1.

Figure1(b) shows the magnetic field dependence of the cross sections for spin relaxation in $\mathrm{He}+\mathrm{CaH}$ collisions in the $s$-wave scattering regime $\left(E_{C}=10^{-5} \mathrm{~cm}^{-1}\right)$. The $\mathrm{CC}$ cross sections grow monotonously with the Zeeman splitting between the $M_{S}= \pm 1 / 2$ levels of CaH due to an enhanced rate of tunnelling through the centrifugal barrier in the outgoing collision channel ${ }^{16}$. The restricted basis set calculations with $K_{\max }=1$ are in excellent agreement with the accurate $\mathrm{CC}$ results regardless the entire range of magnetic field explored here (0.003 T - $1 \mathrm{~T})$. However, the minimal basis set calculations with $K_{\max }=0$ underestimate the inelastic cross sections by 5-7 orders of magnitude

The inability of the minimal basis set $\left(K_{\max }=0\right)$ to provide reliable results for the spin relaxation transition in $\mathrm{CaH}$ indicates that $K=1$ basis functions play an essential role in the spin relaxation mechanism. Indeed, as shown by Krems and co-workers ${ }^{50}$, spin relaxation in collisions of ${ }^{2} \Sigma$ molecules with structureless atoms is a third-order process mediated by the anisotropy of the atom-molecule interaction potential and by the spin-rotation interaction within the excited rotational state manifold. The spin-rotation interaction flips the molecular spin projection $\Sigma$ while changing the value of $K$ by one so as to conserve the BF projection of the total angular momentum $\Omega=K+\Sigma$. A proper description of this process thus requires basis functions with $K=1$, which are missing from the minimal $K_{\max }=0$ basis set, leading to inaccurate results. 


\section{B. $\mathrm{Li}+\mathrm{CaH}$ collisions}

Unlike the $\mathrm{He}+\mathrm{CaH}$ just considered, $\mathrm{Li}+\mathrm{CaH}$ is a prototype of a strongly bound, anisotropic collision complex with spin-dependent interactions. Ultracold $\mathrm{Li}(\uparrow)+\mathrm{CaH}(\uparrow)$ collisions in a magnetic field are predominantly elastic ${ }^{22}$ despite the presence of strongly anisotropic interactions, which indicate good prospects for sympathetic cooling of magnetically trapped $\mathrm{CaH}(N=0)$ molecules by ultracold $\mathrm{Li}$ atoms ${ }^{22}$. In the following, we will use arrows to denote the atomic and molecular Zeeman states $\uparrow(\downarrow)$ corresponding to $M_{S_{i}}=+1 / 2(-1 / 2)$. For instance, collisions of $\mathrm{CaH}$ molecules with Li atoms initially in their fully spin-polarized initial states $M_{S_{a}}=M_{S}=1 / 2$ are denoted as $\operatorname{Li}(\uparrow)+\mathrm{CaH}(\uparrow)$.

Figure 2(a) shows the collision energy dependence of the elastic cross section, which approaches a constant value in the $s$-wave regime $\left(E_{C}<10^{-4} \mathrm{~cm}^{-1}\right)$ and displays a shape resonance at $E_{C} \sim 1.5 \times 10^{-3} \mathrm{~cm}^{-1}(2 \mathrm{mK})$. The cross section computed using the maximally truncated basis $\left(K_{\max }=0\right)$ agrees well with full CC results over a wide range of collision energies spanning four orders of magnitude. The agreement is particularly good in the multiple partial-wave regime near the shape resonance, which suggests that single-channel shape resonances can be described with quantitative accuracy by CC calculations including only the lowest value of $K=0$ in the basis set. As Table II shows, such calculations are nearly 90 times more computationally efficient than the full CC calculations.

Figures 2(b)-(d) show the collision energy dependence of the inelastic cross sections calculated at several representative magnetic fields. While at the lowest field of $1 \mathrm{G}$ the cross sections calculated with the minimal basis set $\left(K_{\max }=0\right)$ are indistinguishable from exact $\mathrm{CC}$ results, significant deviations are observed in the intermediate-field regime $(B=100$ $\mathrm{G})$. In particular, $K_{\max }=1$ calculations strongly overestimate the full CC results in the vicinity of the $2 \mathrm{mK}$ shape resonance. As expected, the results computed with restricted basis sets gradually converge to the full CC limit with increasing $K_{\max }$, although as illustrated in Fig. 2(b) the convergence can be slow. We attribute this to the presence of a scattering resonance in the inelastic cross section, which occurs in the multiple partial-wave regime near $B=100 \mathrm{G}$. As shown below, the resonance is mediated by the spin-rotation and the centrifugal couplings, which connect basis functions with $K^{\prime}=K \pm 1$, and the matrix elements of the interaction potential, which is diagonal in $K$.

As shown in our previous work $^{22}$, spin relaxation in doubly polarized $\mathrm{Li}(\uparrow)+\mathrm{CaH}(\uparrow)$ 
collisions occurs through an interplay of two mechanisms. The first indirect mechanism discussed in Sec. IIB above involves the spin-rotation interaction in the excited rotational states of $\mathrm{CaH}$ and the anisotropy of the $\mathrm{Li}-\mathrm{CaH}$ interaction potential. The second mechanism is a direct relaxation process mediated by the magnetic dipole-dipole interaction 24,26 between $\mathrm{Li}(\uparrow)$ and $\mathrm{CaH}(\uparrow)$. Because the magnetic dipole-dipole interaction is diagonal in $K$ (see, e.g. Eq. (8) of Ref. 26) the direct mechanism does not require a change in the value of $K$, in contrast to the indirect mechanism discussed above, which does. The rapid convergence of restricted basis set calculations to the exact result in the ultracold regime shown in Fig. 2(b)(d) is then due to the dominance of the direct spin relaxation mechanism in this regime ${ }^{22}$.

Figure 3 shows the magnetic field dependence of the inelastic cross sections for $\mathrm{Li}(\uparrow)+\mathrm{CaH}(\uparrow)$ collisions at two representative collision energies. In the $s$-wave regime $\left(E_{C}=10^{-6} \mathrm{~K}\right)$, dominated by a single $l=0$ incident partial wave, the cross sections increase at low magnetic fields, reach a minimum near $B=20 \mathrm{G}$ and continue to increase at higher magnetic fields. The restricted basis set calculations with $K_{\max }=0$ are in excellent agreement with full CC results over the entire range of magnetic fields.

A different trend is observed in the multiple partial wave regime $\left(E_{C}=10^{-3} \mathrm{~K}\right)$ in Fig. 3 (b). While the inelastic cross section displays a smooth magnetic field dependence below $50 \mathrm{G}$, a resonance pattern is observed at higher magnetic fields. The figure shows that the convergence with respect to $K_{\max }$ slows down at higher magnetic fields, and especially in the vicinity of scattering resonances. Restricted basis set calculations using small basis sets do not reproduce the details of the resonance structure, which requires basis sets with $K_{\max } \geq 3$, at which point restricted basis set calculations become nearly as computationally expensive as full CC calculations (see Table II).

The slow convergence with respect to $K_{\max }$ indicates that the matrix elements between the $K$-states omitted from the basis set are essential for the proper description of the resonance structure in Fig. 3(b). As discussed at the end of Sec. IIC, these matrix elements can involve either (1) the spin-rotation interaction (Eq. 7), (2) the magnetic dipole-dipole interaction, (3) the atom-molecule interaction potential (Eq. 8), and (4) the squared orbital angular momentum operator (or the centrifugal term, see Eq. 9).

To identify which of these terms is responsible for the resonance structure shown in Fig. 3(b), we carried out test calculations omitting the matrix elements of the specific terms (1)-(4) one by one. Figure 4(b) shows that neglecting the matrix elements of the magnetic 
dipole-dipole interaction between the basis states with $K=3$ has little effect on the resonance profile of the inelastic cross section. In contrast, as shown in Figs. 4(a), (c) and (d), omitting the matrix elements of the spin-rotation, interaction potential, or centrifugal terms between the basis states with $K=3$ leads to a dramatic change of the resonance profiles. This indicates that all of these interactions play an essential role in determining the resonance structure. Particularly crucial are the diagonal matrix elements of the interaction potential between $K=3$ basis functions.

The error due to the truncation of the helicity basis set may be further subdivided into the errors due to the neglect of the diagonal and off-diagonal matrix elements between the basis functions with $K>K_{\max }$ of the spin-rotation and centrifugal interactions. To eliminate the diagonal error for these terms, we performed additional calculations using a complete helicity basis set but neglecting the off-diagonal couplings between the states of different $K$, thereby invoking the helicity-conserving CS approximation $\stackrel{33,34}{ }$. The results are shown in Fig. 5. We observe that the resonance profiles are sensitive to the off-diagonal matrix elements of the spin-rotation interaction and those of the centrifugal term. Remarkably, while omitting the off-diagonal matrix elements of the spin-rotation interaction splits the resonance peak in two narrower peaks, neglecting the Coriolis coupling completely sweeps out the resonance profile as seen in Fig. 5(b). This is in line with a previous CS study of the chemical reaction $\mathrm{F}+\mathrm{HCl}$, which demonstrated the inability of the $\mathrm{CS}$ approximation to describe the resonance structure in low-energy reaction cross sections $\frac{37}{2}$. We conclude that a fully quantitative description of the magnetic resonance structure in $\mathrm{Li}+\mathrm{CaH}$ spin relaxation collisions requires not only a nearly-complete helicity basis, but also the proper inclusion of all couplings between the different $K$ states in the scattering Hamiltonian.

\section{C. $\mathrm{Li}+\mathrm{SrOH}$ collisions}

Thus far we have applied helicity basis truncation to ultracold collisions of S-state atoms with a light molecule $(\mathrm{CaH})$. The spectrum of rotational levels of $\mathrm{CaH}$ is relatively sparse compared to that of the heavier molecular radicals slowed and trapped in recent experiments, such as $\mathrm{SrOH}^{53}$ and $\mathrm{SrF}^{54}, 55$. To investigate the performance of restricted helicity basis sets for collisions of heavy molecules with a dense rotational spectrum, we consider ultracold $\mathrm{Li}(\uparrow)+\mathrm{SrOH}(\uparrow)$ collisions in a magnetic field. The ground ${ }^{2} \Sigma$ electronic state of $\mathrm{SrOH}$ has 
been Sisyphus cooled ${ }^{53}$ and our recent $\mathrm{CC}$ study of $\mathrm{Li}+\mathrm{SrOH}$ collisions has established good prospects of sympathetic cooling of $\mathrm{SrOH}(\uparrow)$ with $\mathrm{Li}(\uparrow)$ atoms in a magnetic trap 24 .

Figure 6 compares the elastic and inelastic cross sections for $\mathrm{Li}(\uparrow)+\mathrm{SrOH}(\uparrow)$ collisions calculated using truncated basis sets with the full $\mathrm{CC}$ results. As in the cases of $\mathrm{He}+\mathrm{CaH}$ and $\operatorname{Li}(\uparrow)+\mathrm{CaH}(\uparrow)$ collisions considered above, the elastic cross sections obtained using the minimal basis set $\left(K_{\max }=0\right)$ are in excellent agreement with full CC results over the entire range of collision energies from $10^{-3} \mathrm{~cm}^{-1}$ down to the s-wave regime. At low magnetic fields, the same is true for the inelastic cross sections shown in Fig. 6(b). However, the situation changes when the magnetic field is increased: As shown in Fig. 6(c), minimalbasis-set results overestimate the accurate CC cross section by a factor of 2-3 depending on the collision energy. Obtaining fully converged results at higher magnetic fields $(B=1000 \mathrm{G})$ requires larger helicity basis sets with $K_{\max } \geq 2$. The computational gain achieved in this case is modest (see Table I).

Figure 7 shows the magnetic field dependence of the cross sections for spin relaxation in ultracold $\mathrm{Li}(\uparrow)+\operatorname{SrOH}(\uparrow)$ collisions in the $s$-wave regime. The cross sections increase monotonously with magnetic field at low fields $(B<500 \mathrm{G})$ and display a resonance structure at higher fields. The background values of the inelastic cross sections at low fields can be accurately described with $K_{\max } \leq 1$ basis sets at only a small fraction of the computational cost of the full CC calculations (see Table I). Reproducing the details of the resonance structure in the inset of Fig. 7 requires larger basis sets $K_{\max } \geq 2$, suggesting the importance of the rotational and Coriolis interactions in determining the resonance structure (see Sec. IIIB above).

It is instructive to compare the overall performance of restricted helicity basis sets applied to $\mathrm{Li}+\mathrm{SrOH}$ vs $\mathrm{Li}+\mathrm{CaH}$ collisions. Despite the very disparate rotational constants of $\mathrm{CaH}$ $\left(B=4.2 \mathrm{~cm}^{-1}\right)$ and $\mathrm{SrOH}\left(B=0.25 \mathrm{~cm}^{-1}\right)$ the Li-SrOH and Li-CaH collision complexes have similar reduced masses and hence similar Coriolis interactions, leading one to expect no significant differences in performance. Comparing Fig. 2 and Fig. 6 we see that this is indeed the case: In both cases relatively large basis sets with $K_{\max } \geq 2$ are required to properly describe the resonance structure at high magnetic fields. This observation suggests that restricted basis set calculations might provide more accurate results for heavier atommolecule collisions ( $\mathrm{such}$ as $\mathrm{Rb}+\mathrm{SrF}^{26}$ ) where the Coriolis interaction is suppressed by the large reduced mass of the collision complex. 


\section{SUMMARY AND OUTLOOK}

We have explored the use of truncated helicity basis sets to enhance the computational efficiency of quantum scattering calculations of ultracold atom-molecule collisions in the presence of an external magnetic field. We have demonstrated that accurate elastic and inelastic cross sections for cold $\mathrm{He}+\mathrm{CaH}, \mathrm{Li}+\mathrm{CaH}$, and $\mathrm{Li}+\mathrm{SrOH}$ collisions can be obtained with a limited number of $K$-states in the basis set. This leads to substantial, 10-100 fold gains in computational efficiency over the full CC calculations (see Table II) for heavy, strongly anisotropic atom-molecule collision complexes such as Li-CaH and Li-SrOH.

The rate of convergence of restricted basis set calculations towards the exact (full CC) results is an important factor that determines the computational advantage of using truncated helicity basis sets. We found that this rate generally depends on the collision system under consideration, the collision energy and on the magnitude of the external magnetic field. The fastest convergence is observed for elastic cross sections: As shown in Figs. 1, 2, and 6, quantitative accuracy can be achieved away from the resonance region with a minimal basis set $\left(K_{\max }=0\right)$ over wide range of collision energies for all of the collision systems studied here. The spin relaxation cross sections for $\mathrm{Li}+\mathrm{CaH}$ and $\mathrm{Li}+\mathrm{SrOH}$ in the $s$-wave regime also display extremely rapid convergence with respect of $K_{\max }$ as shown in Figs. 3)(a) and 7. In these regime, we achieve computational gains on the order of 10-100 (see Table II). The reason for such rapid convergence is that the main mechanisms responsible for either elastic scattering or dipolar-medicated spin relaxation in $\mathrm{Li}+\mathrm{CaH}$ and $\mathrm{Li}+\mathrm{SrOH}$ collisions are diagonal in $K$. Larger basis sets are required to describe collisional relaxation mechanisms that do not conserve $K$, such as the intramolecular spin-rotation interaction, which is responsible for spin relaxation in $\mathrm{He}+\mathrm{CaH}$ collisions [Fig. 1(b)].

Another instance where the $K$ non-conserving mechanisms are important is provided by the magnetic resonance structure in $\mathrm{Li}+\mathrm{CaH}$ and $\mathrm{Li}+\mathrm{SrOH}$ collisions in the few-partial wave regime [Figs. 3(b) and 7]. These resonances arise as a result of an interplay between the atom-molecule interaction potential, the spin-rotation interaction, and the centrifugal interaction, which have both the diagonal and off-diagonal matrix elements in $K$ (except for the interaction potential). Excluding the basis functions with $K \geq K_{\max }$ from the basis

eliminates the essential matrix elements from the matrix representation of the Hamiltonian, leading to either a substantial modification (for small $K_{\max }$ ) or complete disappearance (for 
$\left.K_{\max }=0\right)$ of the resonance structure as shown in Figs. B(b) and 7 . We conclude that the accurate description of the resonance structure as a function of either collision energy or magnetic field requires nearly complete helicity basis sets with $K_{\max }$ approaching the maximal possible values of $J$ included in the basis set.

However, if the accurate description of the resonance positions and widths is not required, the background values of the inelastic cross sections can be estimated in calculations employing minimal basis sets [see Figs. 3(b) and 7.]

In summary, our results demonstrate that $\mathrm{CC}$ calculations employing restricted helicity basis sets can be used to obtain accurate atom-molecule scattering cross sections over a wide range of collision energies and magnetic fields at a fraction of the computational cost of the fully rigorous CC calculations (see Table II). However, this technique converges much more slowly in the presence of scattering resonances, and hence must be used with caution when applied to calculate the resonance positions and widths.

In future work, it would be interesting to explore the performance of truncated helicity basis sets in $\mathrm{CC}$ calculations of heavy atom-molecule collisions (such as Rb-SrF $\stackrel{26}{ }$ ). The weaker Coriolis interactions in these systems may lead to better performance of truncated basis sets. This methodology may also be applied to the computationally challenging problems of atom-molecule reactive scattering or molecule-molecule collisions in external electromagnetic fields $\underline{14}, \underline{56}$.

\section{ACKNOWLEDGEMENTS}

We are grateful to Naduvalath Balakrishnan for a helpful discussion and to Roman Krems for providing access to additional computational resources. This work was supported by NSF grant No. PHY-1607610. 
*ttscherbul@unr.edu

1 R. V. Krems, W. C. Stwalley, and B. Friedrich, Cold Molecules: Theory, Experiment, Applications (CRC press, Taylor \& Francis, 2009).

2 O. Dulieu and A. Osterwalder, Cold Chemistry: Molecular Scattering and Reactivity Near Absolute Zero (RSC, 2017).

3 J. L. Bohn, A. M. Rey, and Jun Ye, Science 357, 1002 (2017).

4 L. D. Carr, D. DeMille, R. V. Krems, and J. Ye, New J. Phys. 11, 055049 (2009).

5 S. Ospelkaus, K.-K. Ni, D. Wang, M. H. G. de Miranda, B. Neyenhuis, G. Quéméner, P. S. Julienne, J. L. Bohn, D. S. Jin, and J. Ye, Science 327, 853 (2010).

6 K.-K. Ni, S. Ospelkaus, D. Wang, G. Quéméner, B. Neyenhuis, M. H. G. de Miranda, J. L. Bohn, J. Ye, and D. S. Jin, Nature 464, 1324 (2010).

7 B. K. Stuhl, M. T. Hummon, and J. Ye, Annu. Rev. Phys. Chem. 65, 501 (2014).

8 X, Wu, T. Gantner, M. Koller, M. Zeppenfeld, S. Chervenkov, G. Rempe, Science 358, 645 (2017).

9 P. Casavecchia and M. H. Alexander, Science 341, 1076 (2013).

10 S. Chefdeville, Y. Kalugina, S. Y. T. van de Meerakker, C. Naulin, F. Lique, M. Costes, Science 341, 1094 (2013).

11 E. Lavert-Ofir, Y. Shagam, A. B. Henson, S. Gersten, J. Kłos, P. S. Żuchowski, J. Narevicius, and E. Narevicius, Nat. Chem. 6, 332 (2014).

12 N. Balakrishnan and A. Dalgarno, Chem. Phys. Lett. 341, 652 (2001); 351, 159 (2002).

13 N. Balakrishnan, J. Chem. Phys. 145, 150901 (2016).

14 T. V. Tscherbul and R. V. Krems, Phys. Rev. Lett. 115, 023201 (2015).

15 R. V. Krems and A. Dalgarno, J. Chem. Phys. 120, 2296 (2004).

16 A. Volpi and J. L. Bohn, Phys. Rev. A 65, 052712 (2002).

17 T. V. Tscherbul and R. V. Krems, Phys. Rev. Lett. 97, 083201 (2006).

18 M. L. González-Martínez and J. M. Hutson, Phys. Rev. A 75, 022702 (2007).

19 R. V. Krems, Phys. Chem. Chem. Phys. 10, 4079 (2008).

20 T. V. Tscherbul, in Cold Chemistry: Molecular Scattering and Reactivity Near Absolute Zero, Chap. 6, p. 276 (ed. by A. Osterwalder and O. Dulieu, RSC, 2017). 
21 M. Lara, J. L. Bohn, D. Potter, P. Soldan, and J. M. Hutson, Phys. Rev. Lett. 97, 183201 (2006).

22 T. V. Tscherbul, J. Kłos, and A. A. Buchachenko, Phys. Rev. A 84, 040701(R) (2011).

23 J. Lim, M. D. Frye, J. M. Hutson, and M. R. Tarbutt, Phys. Rev. A 92, 053419 (2015).

24 M. Morita, J. Kłos, A.A. Buchachenko, and T. V. Tscherbul, Phys. Rev. A 95, 063421 (2017).

25 T. V. Tscherbul and A. Dalgarno, J. Chem. Phys. 133, 184104 (2010).

26 M. Morita, M. Kosicki, P. S. Zuchowski, and T. V. Tscherbul, arXiv:1801.07978.

27 T. V. Tscherbul, Phys. Rev. A 85, 052710 (2012).

28 J. M. Launay and M. Le Dourneuf, Chem. Phys. Lett. 169, 473 (1990).

29 B. Lepetit and J. M. Launay, J. Chem. Phys. 95, 5159 (1991).

30 R. V. Krems and S. Nordholm, J. Chem. Phys. 115, 10581 (2001).

31 J. Hazra and N. Balakrishnan, New J. Phys. 17, 055027 (2015).

32 D. Skouteris, J. F. Castillo, and D. E. Manolopoulos, Comp. Phys. Commun. 133, 128 (2000).

33 R. T Pack, J. Chem. Phys. 60, 633 (1974).

34 P. McGuire and D. J. Kouri, J. Chem. Phys. 60, 2488 (1975).

35 R. V. Krems, J. Chem. Phys. 116, 4525 (2002).

36 J. Kłos and F. Lique, in Cold Chemistry: Molecular Scattering and Reactivity Near Absolute Zero, Chap. 2, p. 46 (ed. by A. Osterwalder and O. Dulieu, RSC, 2017).

37 G. Quéméner and N. Balakrishnan, J. Chem. Phys. 128, 224304 (2008).

38 A. van der Avoird, P. E. S. Wormer, and R, Moszynski, Chem. Rev. 94, 1931 (1994).

39 R. T Pack and G. A. Parker, J. Chem. Phys. 87, 3888 (1987).

40 M. Mizushima, The Theory of Rotating Diatomic Molecules (Wiley, New York, 1975).

41 J. M. Brown and A. Carrington, Rotational Spectroscopy of Diatomic Molecules (Cambridge University Press. 2003).

42 G. C. Groenenboom, and N. Balakrishnan, J. Chem. Phys. 118, 7380 (2003).

43 M. B. Kosicki, D. Kȩdziera, and P. S. Żuchowski, J. Phys. Chem. A 121, 4152 (2017).

44 T. V. Tscherbul, G. Groenenboom, R. V. Krems, and A. Dalgarno, Faraday Discuss. 142, 127 (2009).

45 T. V. Tscherbul, J. Kłos. L. Rajchel, and R. V. Krems, Phys. Rev. A 75033416 (2007).

46 M. L. González-Martínez and J. M. Hutson, Phys. Rev. A 84, 052706 (2011).

47 D. E. Manolopoulos, J. Chem. Phys. 85, 6425 (1986). 
48 B. R. Johnson, J. Comp. Phys. 13, 445 (1973).

49 R. V. Krems, A. Dalgarno, N. Balakrishnan, and G. C. Groenenboom, Phys. Rev. A 67, 060703 (2003).

50 N. Balakrishnan, G. C. Groenenboom, R. V. Krems, and A. Dalgarno, J. Chem. Phys. 118, $7386(2003)$.

51 J. D. Weinstein, R. deCarvalho, T. Guillet, B. Friedrich, and J. M. Doyle, Nature 395, 148 (1998).

52 V. Singh, K. S. Hardman, M. J. Lu, A. Ellis, M. J. Morrison, and J. D. Weinstein, Mol. Phys. 111, 1711 (2013).

53 I. Kozyryev, L. Baum, K. Matsuda, B. L. Augenbraun, L. Anderegg, A. P. Sedlack, and J. M. Doyle, Phys. Rev. Lett. 118, 173201 (2017).

54 J. F. Barry, D. J. McCarron, E. B. Norrgard, M. H. Steinecker, and D. DeMille, Nature 512, $286(2014)$.

55 D. J. McCarron, M. H. Steinecker, Y. Zhu, and D. DeMille, arXive:1712.01462.

56 Yu. V. Suleimanov, T. V. Tscherbul, and R. V. Krems, J. Chem. Phys. 137, 024103 (2012). 
TABLE I. Convergence parameters of CC calculations (values of $R$ are given in $a_{0}$ ). The reduced masses of the $\mathrm{He}-\mathrm{CaH}$, $\mathrm{Li}-\mathrm{CaH}$, and $\mathrm{Li}-\mathrm{SrOH}$ collision complexes used in $\mathrm{CC}$ calculations were 2.8138538, 5.9902077 and 6.5762047 a.m.u.

\begin{tabular}{cccccc}
\hline \hline System & $R_{\min }$ & $R_{\max }$ & $\Delta R$ & $N_{\max }$ & $J_{\max }$ \\
\hline \hline $\mathrm{He}+\mathrm{CaH}$ & 2.0 & 100.0 & 0.04 & 4 & 4.5 \\
$\mathrm{Li}+\mathrm{CaH}$ & 3.78 & 944.9 & 0.0038 & 55 & 3 \\
$\mathrm{Li}+\mathrm{SrOH}$ & 7.56 & 1133.8 & 0.0019 & 115 & 3 \\
\hline
\end{tabular}


TABLE II. Numbers of scattering channels $N_{c}$ for the full and truncated basis sets. The last column lists the computational gain achieved by using the truncated basis set.

\begin{tabular}{|c|c|c|c|c|c|c|}
\hline System & $J_{\max }$ & $N_{\max }$ & $M$ & $K_{\max }$ & $N_{c}$ & $\left(N_{c}^{\text {full }} / N_{c}\right)^{3}$ \\
\hline \multirow[t]{5}{*}{$\mathrm{He}+\mathrm{CaH}$} & 4.5 & 4 & $1 / 2$ & 4 & 190 & 1 \\
\hline & & & & 3 & 184 & 1.10 \\
\hline & & & & 2 & 164 & 1.55 \\
\hline & & & & 1 & 122 & 3.78 \\
\hline & & & & 0 & 50 & 54.87 \\
\hline \multirow[t]{5}{*}{$\mathrm{Li}+\mathrm{CaH}$} & 3 & 55 & 0 & 4 & 3496 & 1 \\
\hline & & & & 3 & 3392 & 1.09 \\
\hline & & & & 2 & 2968 & 1.63 \\
\hline & & & & 1 & 2104 & 4.59 \\
\hline & & & & 0 & 784 & 88.67 \\
\hline \multirow{5}{*}{$\mathrm{Li}+\mathrm{SrOH}$} & 3 & 115 & 0 & 4 & 7336 & 1 \\
\hline & & & & 3 & 7112 & 1.10 \\
\hline & & & & 2 & 6208 & 1.65 \\
\hline & & & & 1 & 4384 & 4.69 \\
\hline & & & & 0 & 1624 & 92.18 \\
\hline
\end{tabular}



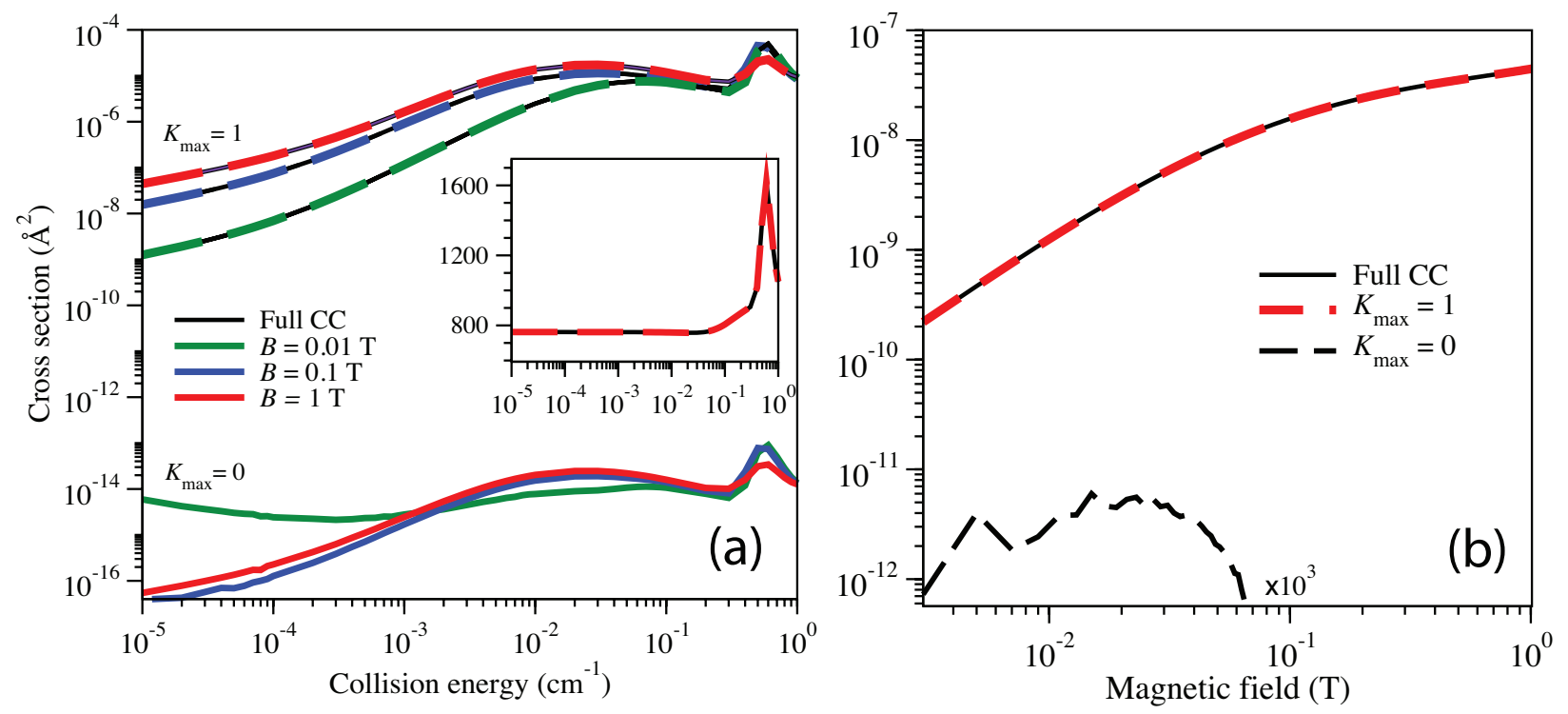

Fig. 1. (a) Spin relaxation cross sections for $\mathrm{He}+\mathrm{CaH}$ collisions as a function of the collision energy. Top lines: full CC results (solid lines) compared with restricted basis set calculations (dashed lines) with $K_{\max }=1$ for $B=1 \mathrm{~T}$ (top), $B=0.1 \mathrm{~T}$ (middle), $B=0.01 \mathrm{~T}$ (bottom). Bottom lines: restricted bases set results for $K_{\max }=0$. The inset shows the elastic cross section as a function of the collision energy at $B=0.01 \mathrm{~T}$. (b) Magnetic field dependence of spin relaxation cross sections for $\mathrm{He}+\mathrm{CaH}$ at $E_{C}=10^{-5} \mathrm{~cm}^{-1}$. Full $\mathrm{CC}$ results (solid line) are compared with restricted basis set results (dashed lines) calculated with $K_{\max }=1$ (red/grey line) and $K_{\max }=0$ (black line). 

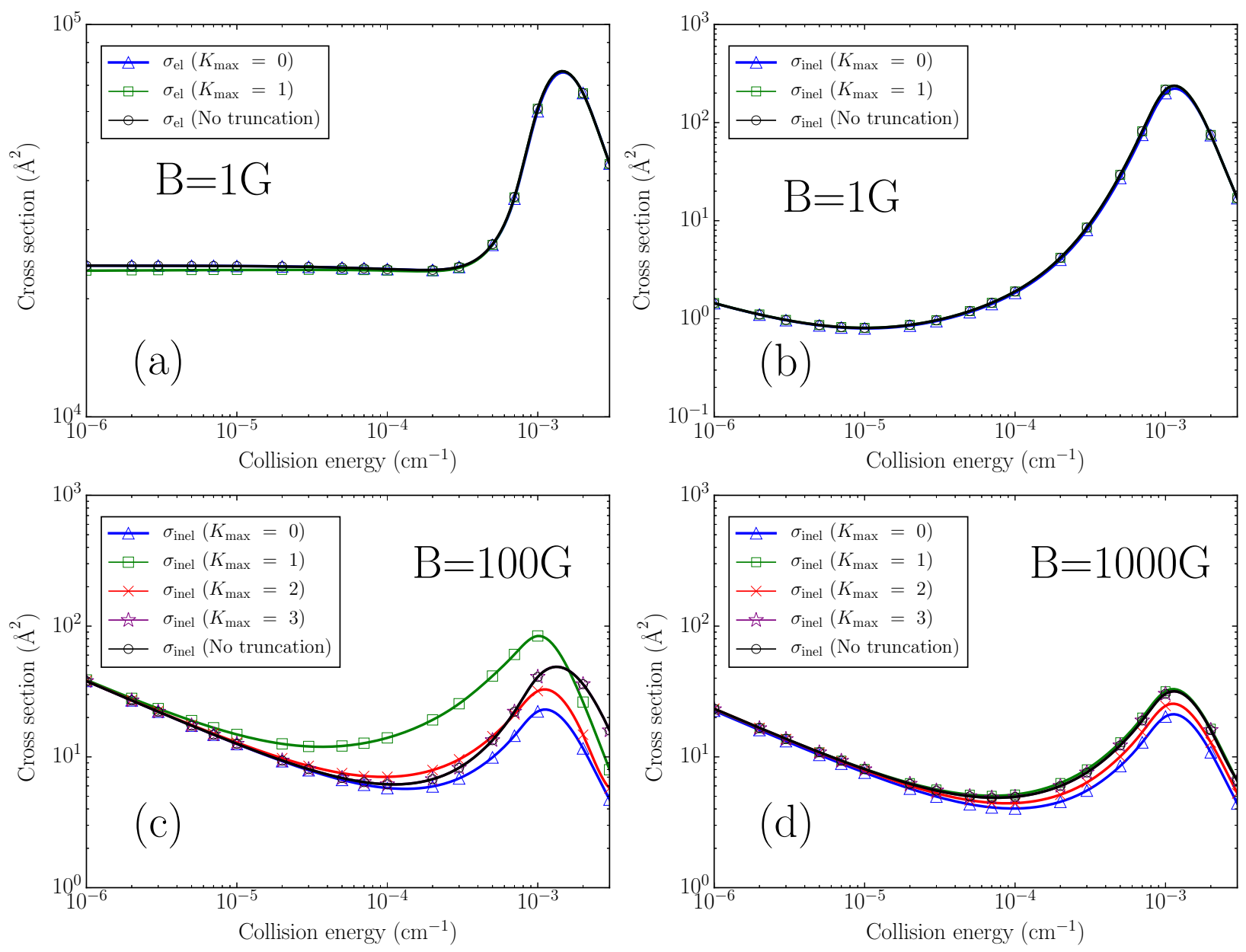

Fig. 2. Elastic (a) and inelastic spin relaxation (b)-(d) cross sections for $\mathrm{Li}(\uparrow)+\mathrm{CaH}(\uparrow)$ collisions as a function of the collision energy for $B=1 \mathrm{G}$ (a)-(b), $B=100 \mathrm{G}$ (c), and $B=1000 \mathrm{G}$ (d). Full $\mathrm{CC}$ results (circles) are compared with restricted basis set calculations with $K_{\max }=0$ (triangles), 1 (squares), 2 (crosses), and 3 (stars). 

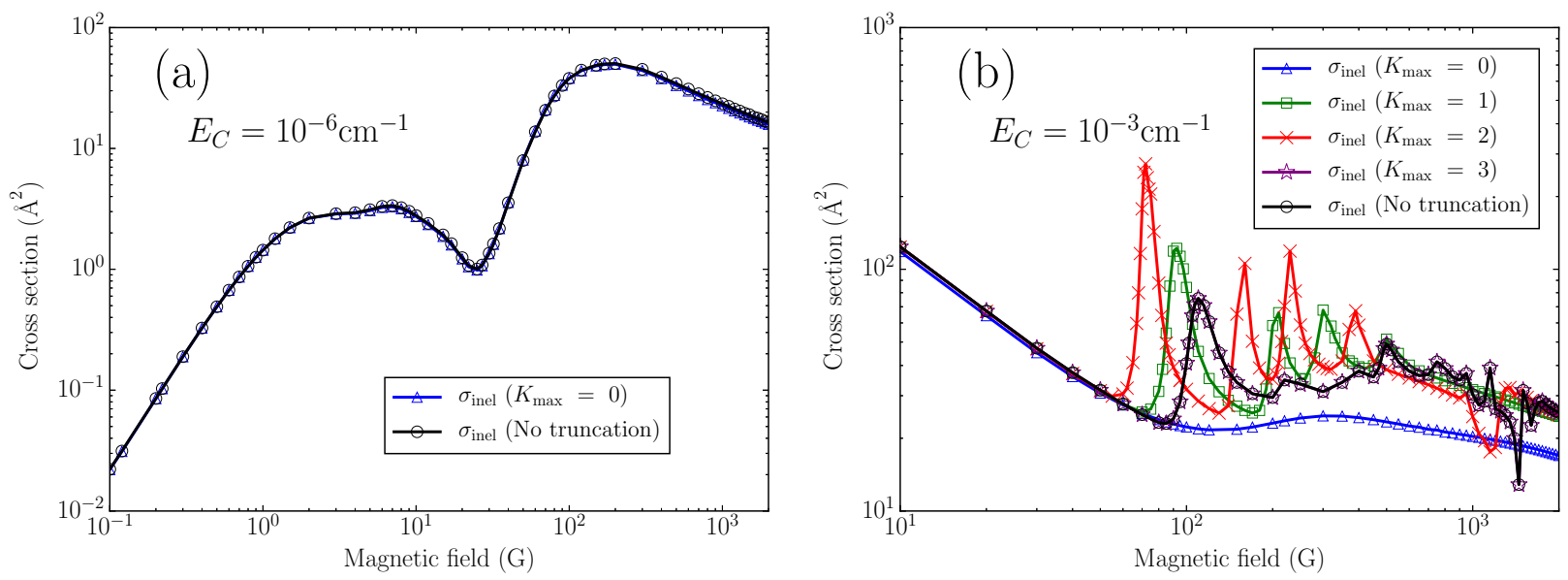

Fig. 3. Magnetic field dependence of spin relaxation cross sections for $\operatorname{Li}(\uparrow)+\mathrm{CaH}(\uparrow)$ collisions at $E_{C}=10^{-6} \mathrm{~cm}^{-1}$ (a) and $E_{C}=10^{-3} \mathrm{~cm}^{-1}$ (b). Full CC results (circles) are compared with restricted basis set calculations with $K_{\max }=0$ (triangles), 1 (squares), 2 (crosses), and 3 (stars). 

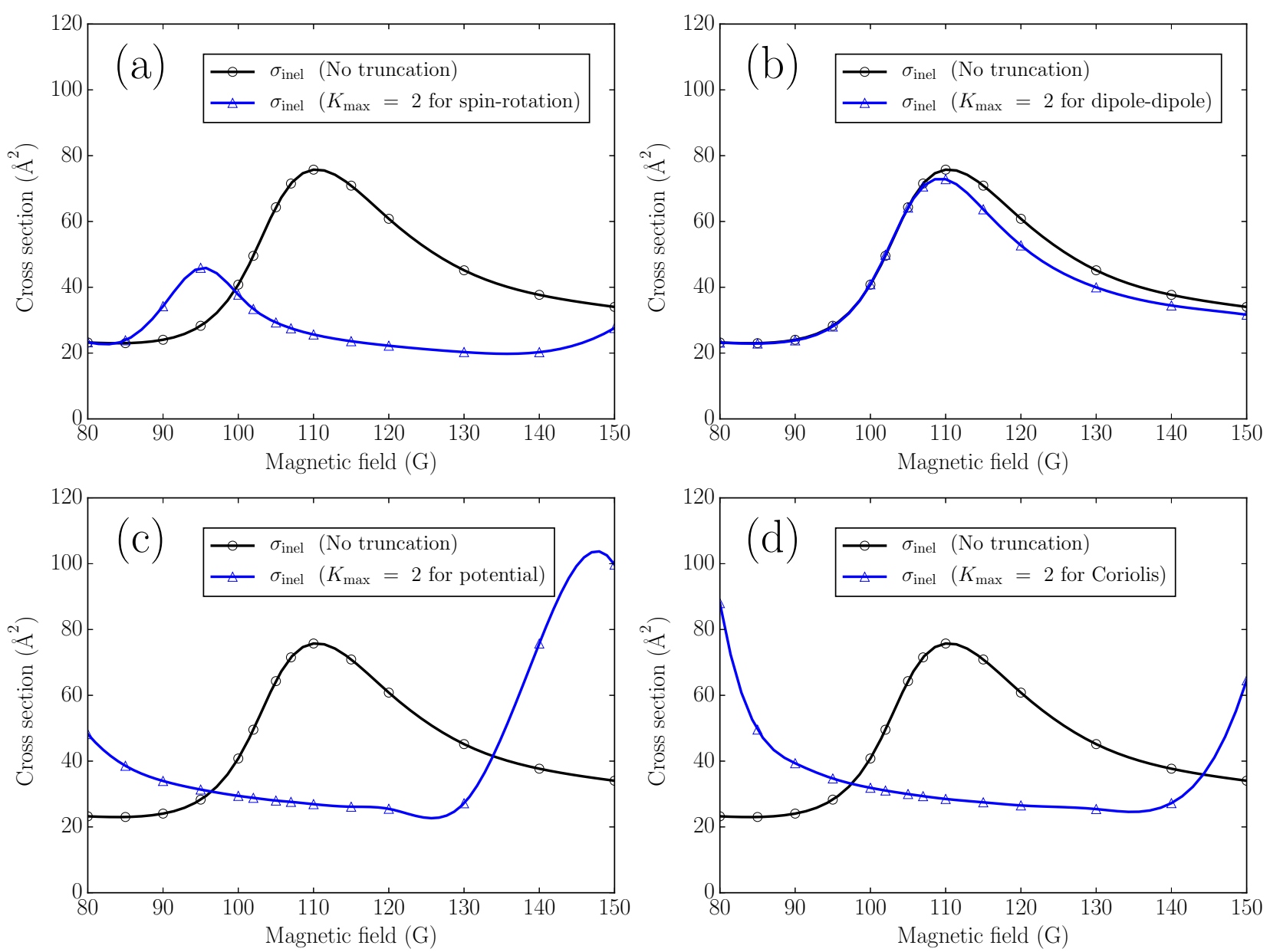

Fig. 4. Magnetic field dependence of spin relaxation cross sections for $\operatorname{Li}(\uparrow)+\mathrm{CaH}(\uparrow)$ collisions at $E_{C}=10^{-3} \mathrm{~cm}^{-1}$ calculated with the $K>K_{\max }=2$ matrix elements of the following terms omitted from the Hamiltonian: (a) spin-rotation interaction, (b) magnetic dipole-dipole interaction, (c) atom-molecule interaction potential, and (d) centrifugal term. Full CC results (circles) are also shown for comparison. 

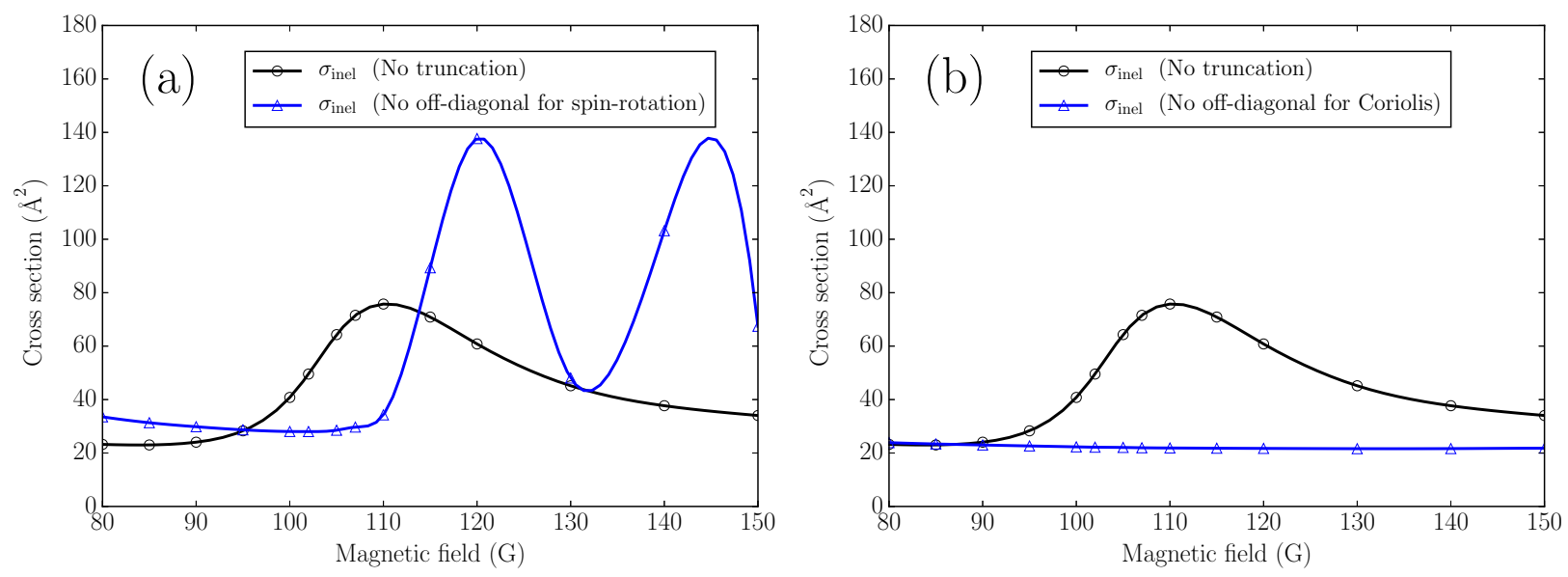

Fig. 5. Magnetic field dependence of spin relaxation cross sections for $\operatorname{Li}(\uparrow)+\mathrm{CaH}(\uparrow)$ collisions at $E_{C}=10^{-3} \mathrm{~cm}^{-1}$ calculated without the off-diagonal in $K$ matrix elements of (a) the spin-rotation interaction and (b) the centrifugal term. Full CC results (circles) are also shown for comparison. 

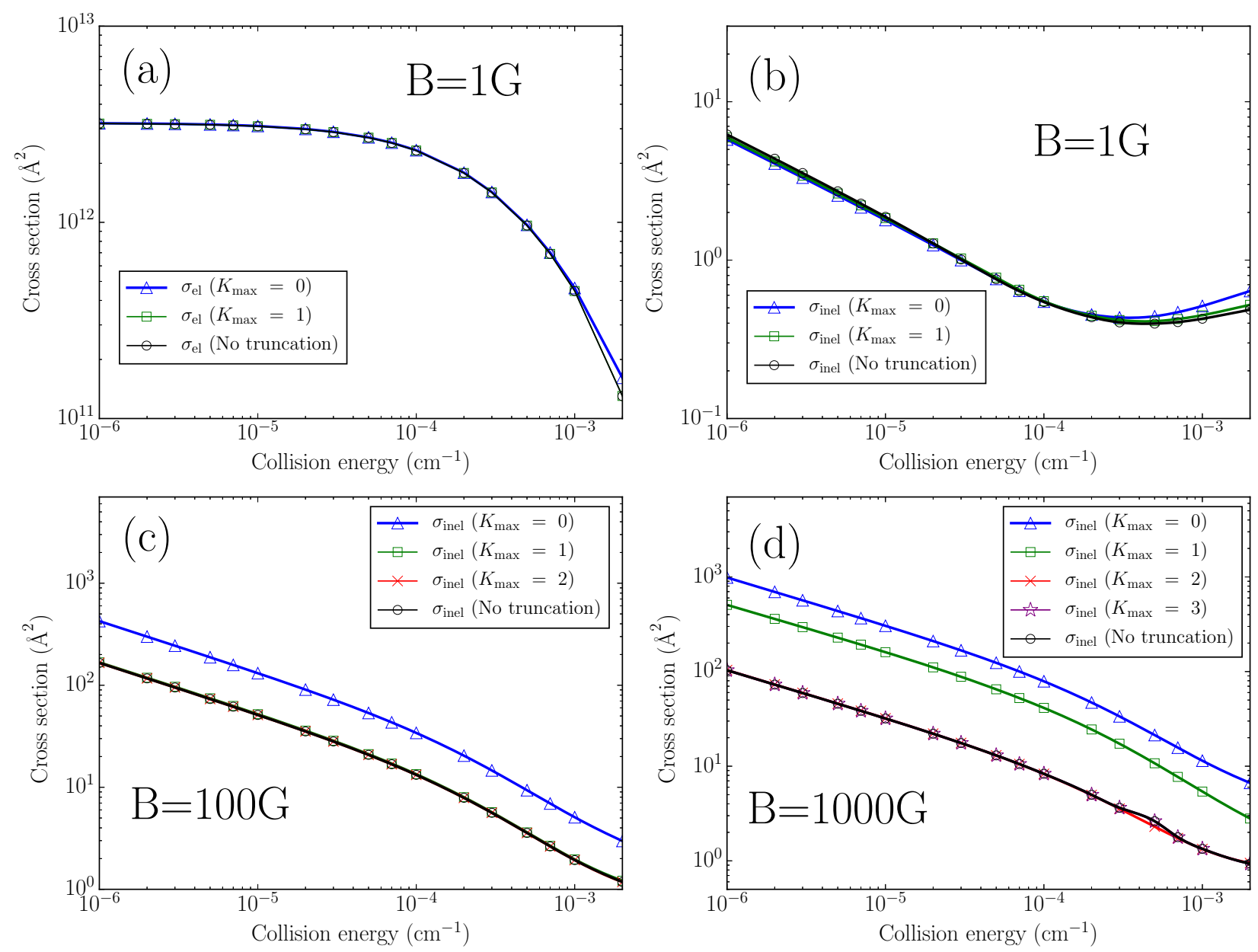

Fig. 6. Elastic (a) and spin relaxation (b)-(d) cross sections for $\operatorname{Li}(\uparrow)+\operatorname{SrOH}(\uparrow)$ collisions as a function of the collision energy for $B=1 \mathrm{G}$ (a)-(b), $B=100 \mathrm{G}$ (c), and $B=1000 \mathrm{G}$ (d). Full $\mathrm{CC}$ results (solid black line) are compared with restricted basis set calculations with $K_{\max }=1$ (squares) and $K_{\max }=0$ (triangles). 


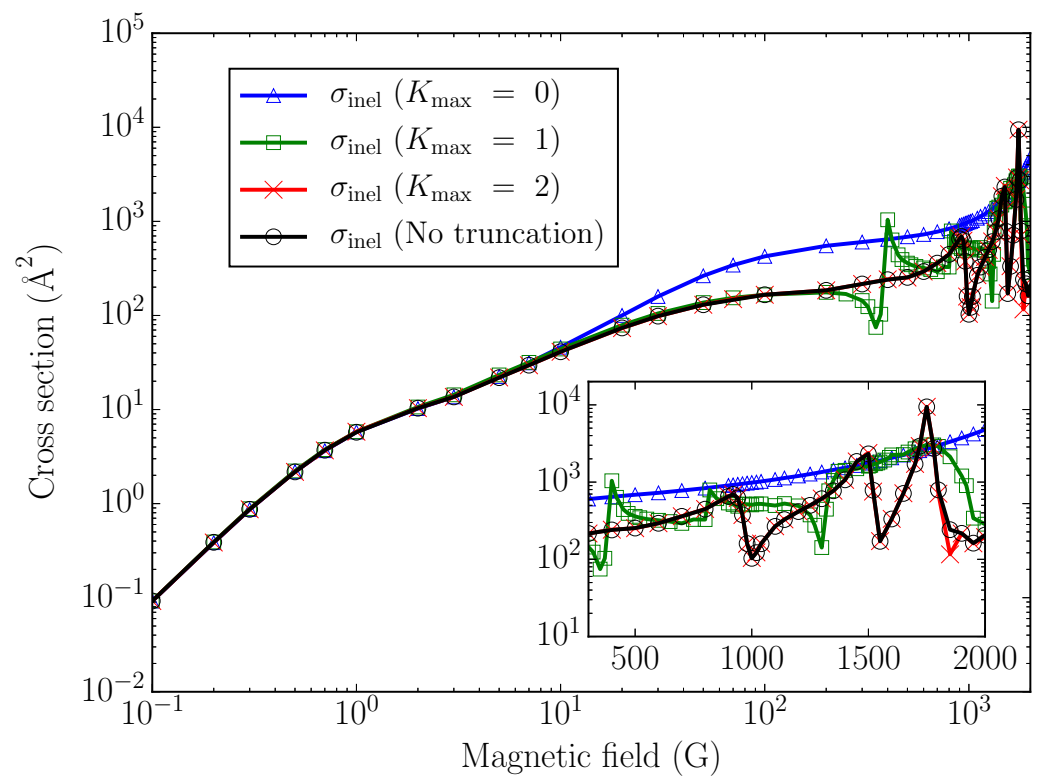

Fig. 7. Magnetic field dependence of spin relaxation cross sections for $\mathrm{Li}(\uparrow)+\mathrm{SrOH}(\uparrow)$ collisions at $E_{C}=10^{-6} \mathrm{~cm}^{-1}$. Full CC results (solid line) are compared with restricted basis set calculations (symbols) with $K_{\max }=0$ (triangles), 1 (squares), 2 (crosses), and 3 (stars). The inset shows the details of the resonance structure at higher magnetic fields. 